\title{
Interpretable instance disease prediction based on causal feature selection and effect analysis
}

YuWen Chen ${ }^{1,2,3}$, Ju Zhang ${ }^{2}$ and XiaoLin Qin ${ }^{1 *}$

\begin{abstract}
Background: In the big wave of artificial intelligence sweeping the world, machine learning has made great achievements in healthcare in the past few years, however, these methods are only based on correlation, not causation. The particularities of the healthcare determines that the research method must comply with the causality norm, otherwise the wrong intervention measures may bring the patients a lifetime of misfortune.

Methods: We propose a two-stage prediction method (instance feature selection prediction and causal effect analysis) for instance disease prediction. Feature selection is based on the counterfactual and uses the reinforcement learning framework to design an interpretable qualitative instance feature selection prediction. The model is composed of three neural networks (counterfactual prediction network, fact prediction network and counterfactual feature selection network), and the actor-critical method is used to train the network. Then we take the counterfactual prediction network as a structured causal model and improve the neural network attribution algorithm based on gradient integration to quantitatively calculate the causal effect of selection features on the output results.
\end{abstract}

Results: The results of our experiments on synthetic data, open source data and real medical data show that our proposed method can provide qualitative and quantitative causal explanations for the model while giving prediction results.

Conclusions: The experimental results demonstrate that causality can further explore more essential relationships between variables and the prediction method based on causal feature selection and effect analysis can build a more reliable disease prediction model.

Keywords: Causal effects, Interpretability, Feature selection, Disease prediction

\section{Background}

Machine learning is becoming an increasingly important tool in healthcare. Some artificial intelligence systems have approached or even surpassed human experts in terms of cancer classification [1], cancer detection [2], diabetic retinopathy detection [3]. Artificial intelligence

\footnotetext{
*Correspondence: keyanche@163.com

${ }^{1}$ Chengdu Institute of Computer Applications, Chinese Academy of Sciences, Chengdu, China

Full list of author information is available at the end of the article
}

(AI) will, without doubt, help reshape the future of medicine.

However, the current methods that have been successfully applied to the above-mentioned medical problems are based only on association rather than causality. In statistics, people acknowledge that association does not logically imply causation $[4,5]$. The relationship between correlation and causation was formalized by Reichenbach [6] as the common cause principle: if two random variables $\mathrm{X}$ and $\mathrm{Y}$ are statistically dependent, then one of the following causal explanations must be hold: (1) $\mathrm{X}$ is the 
direct cause of Y; (2) There is a random variable $Z$, which is the common reason for $\mathrm{X}$ and $\mathrm{Y}$, as shown in Fig. 1. Therefore, compared with association, causality further explores more essential relationships between variables. The core task of causal inference is to reveal the causal relationship between different variables, which enables us to have the following abilities:(1) predict the outcome of a variable after intervention; (2) to estimate the impact of intervention and confounding factors; (3) Enable the model to predict unseen cases. If we think of medical treatment as an intervention and treat effect as an outcome, then these capabilities are needed in healthcare, but most existing approaches do not yet have them. Furthermore the particularities of the healthcare determines that the research method must comply with the causality norm, otherwise the wrong intervention measures may bring the patients a lifetime of misfortune. Therefore, causality plays a key role in developing truly intelligent medical algorithms.

In addition, with the rapid development of modern medical technology, more and more clinical observation data of patients are collected.However, this growth has a huge impact on the disease prediction model and the time consumption of patient detection and testing. In fact, contrary to popular belief, more variables is not

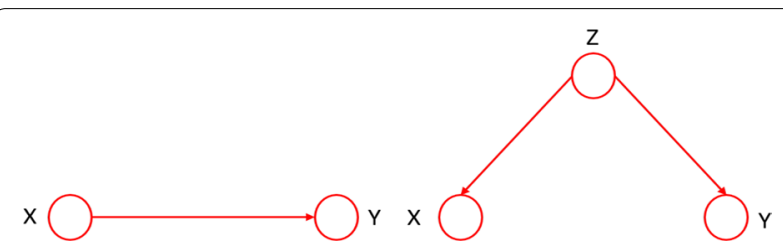

Fig. 1 Causality diagram synonymous of more useful information and a better prediction while in theory the more features are used the better. This can be easily explained by the fact that non relevant features induce over fitting and so decrease the performances and the generalization of the model. The traditional feature extraction can achieve good results in prediction and classification, but it describes the correlation between variables. Therefore, feature selection is one of the important steps to obtain a good prediction effect. In the case of cancer, for example, we need to know what causes it and what variables need to be used to cure it. In lung cancer, both smoking and coughing are contributing factors, but we need to know which the cause is and which the effect is. Because curing cough is not a cure for cancer as a result, banning smoking can prevent cancer because it is a direct cause.

Therefore, we propose a two-stage prediction method (instance feature selection prediction and causal effect analysis) for instance disease prediction, starting from knowledge in the medical field to infer the influence relationship between variables. So as to better understand the underlying mechanism behind the data set and evaluate the model more transparently. The model flow is shown in Fig. 2. Firstly, we use the reinforcement learning framework to design an interpretable qualitative instance feature selection prediction method based on the counterfactual. Then we take the counterfactual prediction network as a structured causal model and improve the neural network attribution algorithm based on gradient integration to quantitatively calculate the causal effect of selection features on the output results.

The main contributions of this paper can be summarized as follows: We use causal mediation analysis for causal feature selection for the first time, and design a

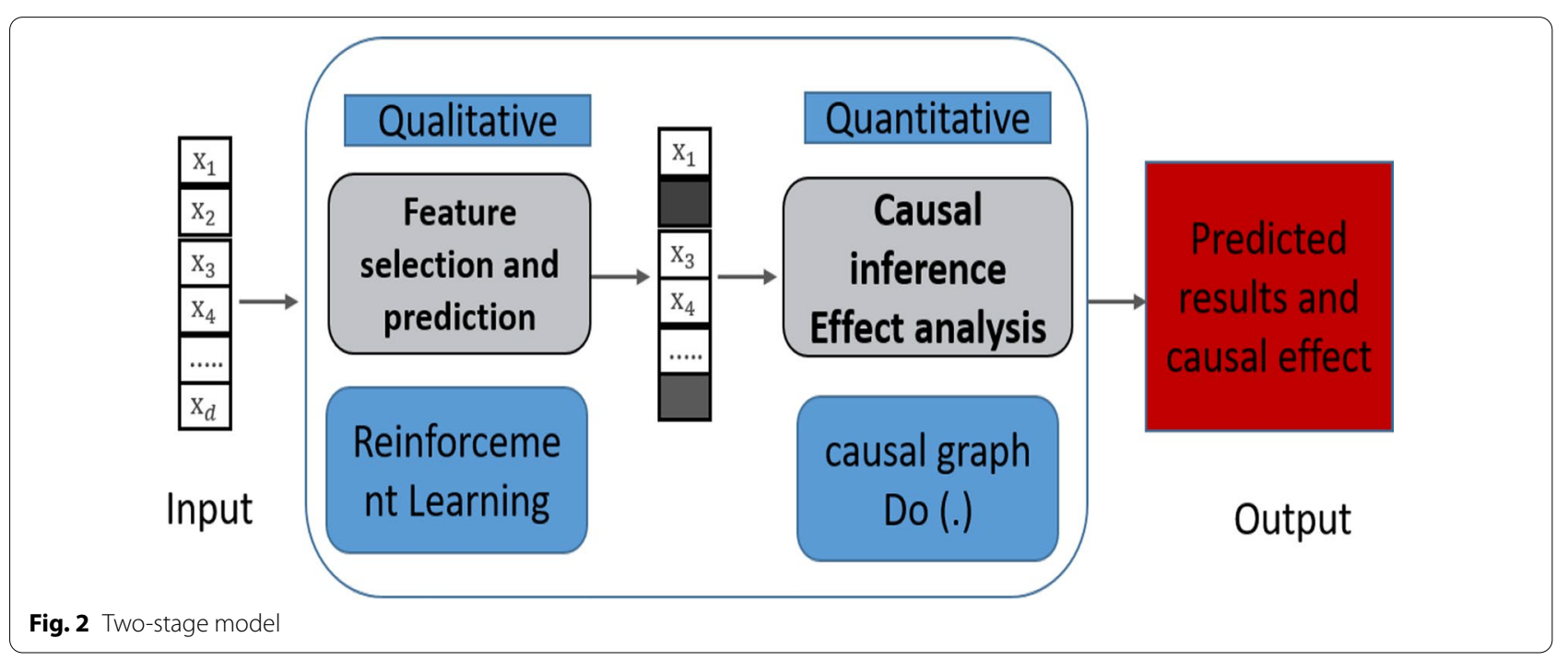


framework for qualitative feature selection based on deep reinforcement learning. In addition, we improve the neural causal attribution algorithm based on the integration gradient, and perform quantitative causal average effect analysis on selected feature attributes in a more robust and interpretable way. Finally, we conducted experimental verification on public data, synthetic data and real medical data, which proved the effectiveness of the method.

\section{Related work}

Machine learning has made great progress in the health [11-13].These apps must satisfy two conditions: (1) they must be causal and (2) they must be explainable. For example, in order to find the effect of a drug on a patient's health, it is necessary to estimate the causal relationship between the drug and the patient's health status. Moreover, in order for the results to be reliable to the doctor, it is necessary to explain how the decision was made.

Recently, interpretability models based on traditional methods have been studied in the following aspects. Attention network: neural network model based on attention mechanism can not only improve the accuracy of prediction, but also specifically show which input features or learning representation are more important for specific prediction, such as graph embedding [14] and machine translation $[15,16]$. Representation learning: One goal of representation learning is to decompose features into independent latent variables that are highly correlated with meaningful patterns [11]. In traditional machine learning, methods such as PCA [17], ICA [18] and spectral analysis [19] are proposed to discover entangled components of data. Recently researchers have developed deep latent variable models such as VAE [20], InfoGan [10] and $\beta$-VAE [21] to learn to untangle the latent variables through variation reasoning. Locally interpretable model: LIME [9] is a representative and precursor framework that can estimate any black box prediction through a local proxy interpretable model. Saliency mapping: Originally developed by Simonyan et al. [22] as a "category saliency map for a particular image", it highlights the pixels of a given input image. These pixels are primarily concerned with identifying a particular category of label for an image. To extract these pixels, a back propagation algorithm can traverse (deconvolution) to find the derivative of the weight vector, and the magnitude of the derivative indicates the importance of each pixel to the category score. Other researchers have used similar concepts to deconvolve predictions and show the location of input images that strongly influence neuronal activation [23-25]. Although these methods are popular tools for interpretability, Adebayo et al. [26] and
Ghorbani et al. [27] argue that relying on visual assessments is insufficient and may be misleading.

In addition, feature selection based on information theory also has corresponding work. Fast correlationbased filter (FCBF) was proposed by Lei Yu and Huan Liu in [33]. This paper mainly proposes to use symmetric uncertainty instead of information gain to measure whether a feature is related to classification $C$ or redundant. Minimum redundancy and maximum relevance (MRMR) algorithm [34] is a feature selection algorithm for single label data. The main purpose of this typical feature attribute selection algorithm is to select $\mathrm{m}$ features from $\mathrm{n}$ features and ensure that the feature subset can keep the classification results of data samples close to or even better than those of all features. Brown et al. [35] present a unifying framework for information theoretic feature selection, bringing almost two decades of research on heuristic filter criteria under a single theoretical interpretation. This paper mainly focuses on the feature selection of causality. Counterfactual analysis and causal inference have gained a lot of attention from the interpretable machine learning field. Research in this area has mainly focused on generating counterfactual explanations from both the data perspective $[28,29]$ as well as the components of a model [30, 31].Pearl [32] introduces different levels of said interpretability and argues that generating counterfactual explanations is the way to achieve the highest level of interpretability. Therefore, this paper attempts to select causal features based on neural network and causal reasoning. The relevant methods are described as follows.

\section{Methods}

The study protocol was approved by the Institutional Ethics Committee of Southwest Hospital of Third Military Medical University (No. KY201936.). We confirm that all methods were performed in accordance with the relevant guidelines and regulations.

In order to provide a common understanding throughout the text, this section describes the concept of Structural Causal Model, Do-operator, and Integral gradient.

\section{Structural causal model (SCM)}

The structural causal model (SCM) [4] is a 4-tuple $\left(\mathrm{X}, \mathrm{U}, \mathrm{f}, P_{u}\right)$, in which $\mathrm{X}$ is a set of finite endogenous variables, usually observable random variables in the system. $\mathrm{U}$ is a finite set of exogenous variables, which are generally regarded as unobserved variables or noise variables. $\mathrm{F}$ is a set of functions $\left[f_{1}, f_{2}, \ldots f_{n}\right]$, where $\mathrm{n}$ refers to the cardinality of the set $X$. These functions define the causal mechanism, such as $\forall x_{i} \in X, x_{i}=f_{i}\left(\right.$ par,$\left.U_{i}\right)$. Par $\in \mathrm{X}-\left\{x_{i}\right\}$ and $U_{i} \in U, P_{u}$ defines the probability distribution on U. Structural causal models represent causal 
dependencies using graphical models that provide an intuitive visualization by representing variables as nodes and relationships between variables as edges in a graph. Graphical models serve as a language for structuring and visualizing knowledge about the world and can incorporate both data-driven and human inputs. Counterfactuals enable the articulation of something there is a desire to know, and structural equations serve to tie the two together.

\section{The do-operator and interventional}

Conditional probability is different from do-operator and intervention distribution. The condition of $\mathrm{T}=\mathrm{t}$ only means that we focus our attention on the people receiving treatment $t$. In contrast, intervention involves treating the entire population. This is illustrated in Fig. 3. We use the do-operator to express intervention: $\mathrm{do}(\mathrm{T}=\mathrm{t})$, which is a commonly used notation in graph causal models and is equivalent to the latent result notation [7]. When the treatment is binary, the average treatment causal effect is as in formula (1):

$$
\mathrm{E}[\mathrm{Y} \mid \operatorname{do}(\mathrm{T}=1)]-\mathrm{E}[\mathrm{Y} \mid \operatorname{do}(\mathrm{T}=0)]
$$

\section{Integral gradient}

Suppose the function $\mathrm{F}: R^{n} \rightarrow[0,1]$ represents a neural network. $\mathrm{x} \in R^{n}$ is the neural network input vector, and $x^{\prime} \in R^{n}$ is the baseline input. Consider the linear path from the baseline $x^{\prime}$ to the input $\mathrm{x}$ in the space $R^{n}$, calculate the gradients of all points along the path, and obtain the integral gradient by accumulating these gradients. Specifically, the integral gradient is defined as the integral path of the gradient along a straight line path from the baseline $x^{\prime}$ to the input $\mathrm{x}$. The integral gradient of input $\mathrm{x}$ and baseline $x^{\prime}$ along the ith dimension is defined as follows, where $\frac{\partial F(x)}{x_{i}}$ is the gradient of $\mathrm{F}(\mathrm{X})$ along the ith dimension.

$$
\begin{aligned}
\operatorname{IntegratedGrad}_{i}(x)= & \left(x_{i}-x_{i}^{\prime}\right) \\
& \times \int_{\alpha=0}^{1} \frac{\partial F\left(x^{\prime}+\alpha \times\left(x-x^{\prime}\right)\right)}{\partial x_{i}} d \alpha
\end{aligned}
$$

\section{Problem formulation}

This work attempts to solve the following problems: "How to achieve qualitative selection of causal features and quantitative causal effect analysis through deep neural networks. That is, how to flexibly select different numbers of causal feature variables for each sample and quantify the causal effects of the selected causal variables on specific output neurons." Therefore, we propose a two-stage causal feature selection prediction and effect analysis method. This is shown in Fig. 2. The details are as follows:

Let $\chi=\chi_{1} \times \chi_{2} \times \ldots \ldots \chi_{d}$ is the $\mathrm{d}$-dimensional feature space, and $\Upsilon=\{1, \ldots, c\}$ is the discrete label space. Let $\mathrm{X}$ represent the collection of all observation attributes of the patient,D $=\left\{\left(X_{i}, Y_{i}\right)\right\}_{i=1}^{n}$ represents a collection of patient clinical data, $X_{i} \in \chi$ Clinical observation data of patient i, $Y_{i} \in \Upsilon$ Ylabel of patient i. Let $\mathrm{Z}$ be a subset of $X$, representing some of the selected dimensional features. Among them, we use the $Z_{\text {opt }}$ to represent the optimal predictive feature set, and $Z_{\sim o p t}$ to represent the non-optimal feature set. Then our problem is to find the optimal $Z_{\text {opt }}$ when predicting the label of each patient, and then analyze the causal effect of the $Z_{\text {opt }}$.

\section{Qualitative causal feature selection}

According to medical knowledge, we can draw the following causality diagram. It can be seen from the Fig. 4 that $\mathrm{Z}$ can be regarded as an mediation variable of $\mathrm{X}$ and $\mathrm{Y}$, which is unobservable and is a hidden variable required by the model.

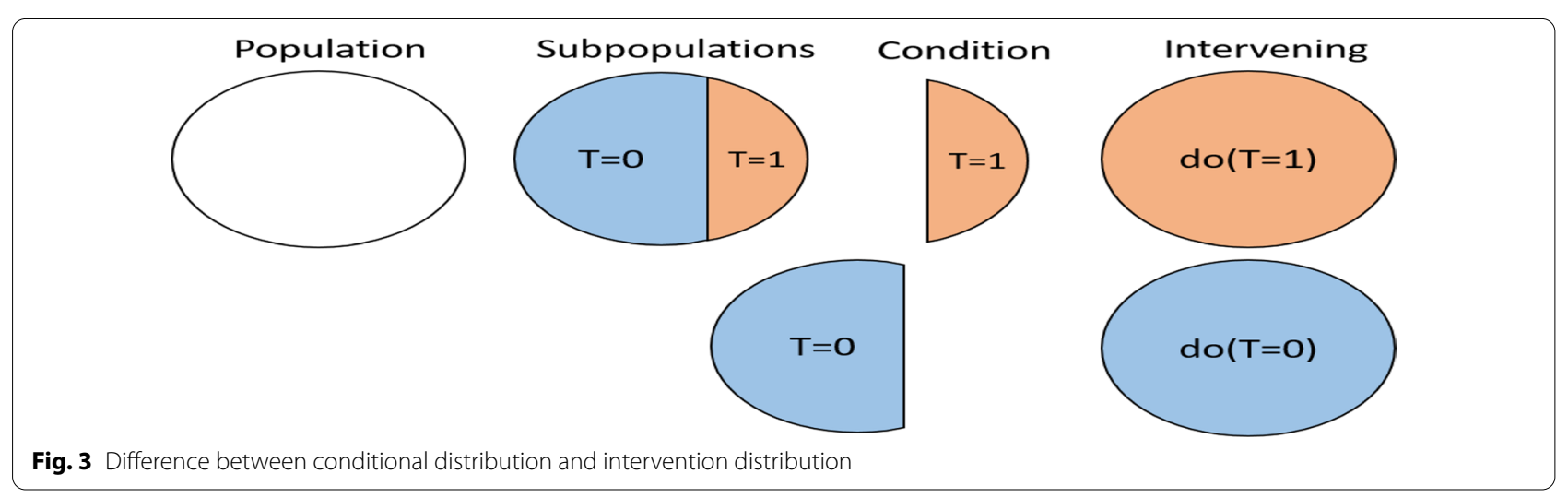




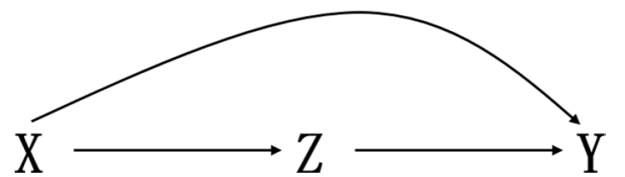

Fig. 4 Causality diagram of patient data. X: observation data set, Z: feature subset, $Y$ target label

If $\mathrm{Z}$ is the optimal predictor subset mediator variable, that is, $\mathrm{Z}$ is required to be completely mediator and the influence of $\mathrm{X}$ on $\mathrm{Y}$ is completely determinable by $\mathrm{Z}$. In other words, it is required to maximize the natural indirect effect (NIE) of formula (3).

$$
\begin{aligned}
\mathrm{NIE}= & \mathrm{P}\left(Y_{Z=z_{o p t}}=1 \mid \operatorname{do}(\mathrm{X}=\mathrm{All})\right) \\
& -\mathrm{P}\left(Y_{Z=Z_{\sim o p t}}=1 \mid \operatorname{do}(\mathrm{X}=\mathrm{All})\right)
\end{aligned}
$$

where $\operatorname{do}(X=A l l)$ means that $X$ takes all the observation attributes set.

The output space size of the feature optimal subset $\mathrm{Z}$ increases exponentially with the size of the feature space. In order to facilitate optimization, we fix $Z_{\sim \text { opt }}$ as the full feature subset $Z_{\sim o p t}=X$ and only intervene $\mathrm{Z}=Z_{\text {opt }}$, Let $\mathrm{Z}$ be a completely mediator, and then minimize formula (4), which is consistent with the definition of relevant feature selection.

$$
N I E^{\prime}=\mathrm{P}\left(Y_{Z=z_{\text {opt }}}=1 \mid \mathrm{X}\right)-\mathrm{P}\left(Y_{Z=X}=1 \mid \mathrm{X}\right)
$$

There is a natural correspondence between interventions in causal reasoning and actions taken in reinforcement learning. Therefore, we define the first half of formula (4) as an actor that performs counterfactual selection prediction on the $Z_{\text {opt }}$. The latter part is defined as a critical, which predicts facts and evaluates actors. We use the Kullback-Leibler (KL) divergence[] to convert constraint (4) into a soft constraint to maximize the causal effect of mediation $\mathrm{Z}$ in formula (5).The model is shown in Fig. 5.

$$
\mathrm{L}(\mathrm{S})=E_{z \sim P z}\left[K L\left(\left(Y_{Z=z_{\text {opt }}} \mid \mathrm{X}\right) \mid\left(Y_{Z=x} \mid \mathrm{X}\right)\right)\right]
$$

Therefore, we use the three neural network to fit the causal structure equation function to optimize the formula (4). $f^{\theta}$ : counterfactual prediction network $\left(Z_{\text {opt }} \rightarrow Y\right), f^{\gamma}$ :fact prediction network $(\mathrm{X} \rightarrow Y), f^{\vartheta}$ : counterfactual selection network $\left(\mathrm{X} \rightarrow Z_{\text {opt }}\right)$.

\section{Counterfactual prediction network}

We design $f^{\theta}$ as a counterfactual predictor network, accepting the selected feature vector of the counterfactual as input, and output the probability distribution on the c-dimensional output space. The loss function of the network is as follows:

$$
l_{1}(\theta)=-E_{(x, y) \sim p_{x y}, z \sim \pi_{\vartheta(x, .)}}\left[\sum_{i=1}^{c} y_{i} \log \left(f_{i}^{\theta}\left(x^{(z)}, z\right)\right)\right]
$$

where $y_{i}$ is the ith component code of $\mathrm{y}$, and $\pi_{\vartheta}$ is the distribution of the counterfactual selection network, which is defined in the next section. $f^{\theta}$ is implemented by a fully connected neural network.

\section{Factual prediction network}

We design $f^{\gamma}$ as the fact prediction network, which is called critical. $f^{\gamma}$ is designed as a fully connected neural network. The network uses all observed patient data to make direct predictions. The loss function of the network is as follows:

$$
l_{2}(\gamma)=-E_{(x, y) \sim p_{x y}},\left[\sum_{i=1}^{c} y_{i} \log \left(f_{i}^{\gamma}(x)\right)\right]
$$

Whether it is a factual prediction network or a counterfactual prediction network, our goal is to make the prediction consistent with the ground truth, and to maximize the probability of choosing the real optimal subset $Z$. Therefore, we fix $\theta, \gamma$, and define the total loss function of the two networks as:

$$
\widehat{l}(x, z)=-\left[\sum_{i=1}^{c} y_{i} \log \left(f_{i}^{\theta}\left(x^{(z)}, z\right)\right)-\sum_{i=1}^{c} y_{i} \log \left(f_{i}^{\gamma}(x)\right)\right]
$$

\section{Counterfactual selection network}

We design $f^{\vartheta}$ as the fact counterfactual selection network. $f^{\vartheta}: \mathrm{X} \rightarrow\{0,1\}^{d}$, The network outputs the selection probability of each feature. The probability of a given feature selection vector $s \in\{0,1\}^{d}$ is:

$$
\pi_{\vartheta}(x, z)=\Pi_{i=1}^{d} f_{i}^{\vartheta}(x)^{s_{i}}\left(1-f_{i}^{\vartheta}(x)\right)^{1-s_{i}}
$$

Define the loss function of the counterfactual selection network:

$$
l_{3}=E_{(x, y) \sim p_{x y}}\left[\sum_{s \in(0,1)^{d}} \pi_{\vartheta}(x, z)\left(\widehat{l}(x, z)+\lambda\left\|f^{\vartheta}\right\|_{0}\right)\right]
$$

We can use the BP back propagation algorithm to train the three neural networks end-to-end, by combining the above three loss functions as shown in Fig. 5. We input patient observation data into the trained 


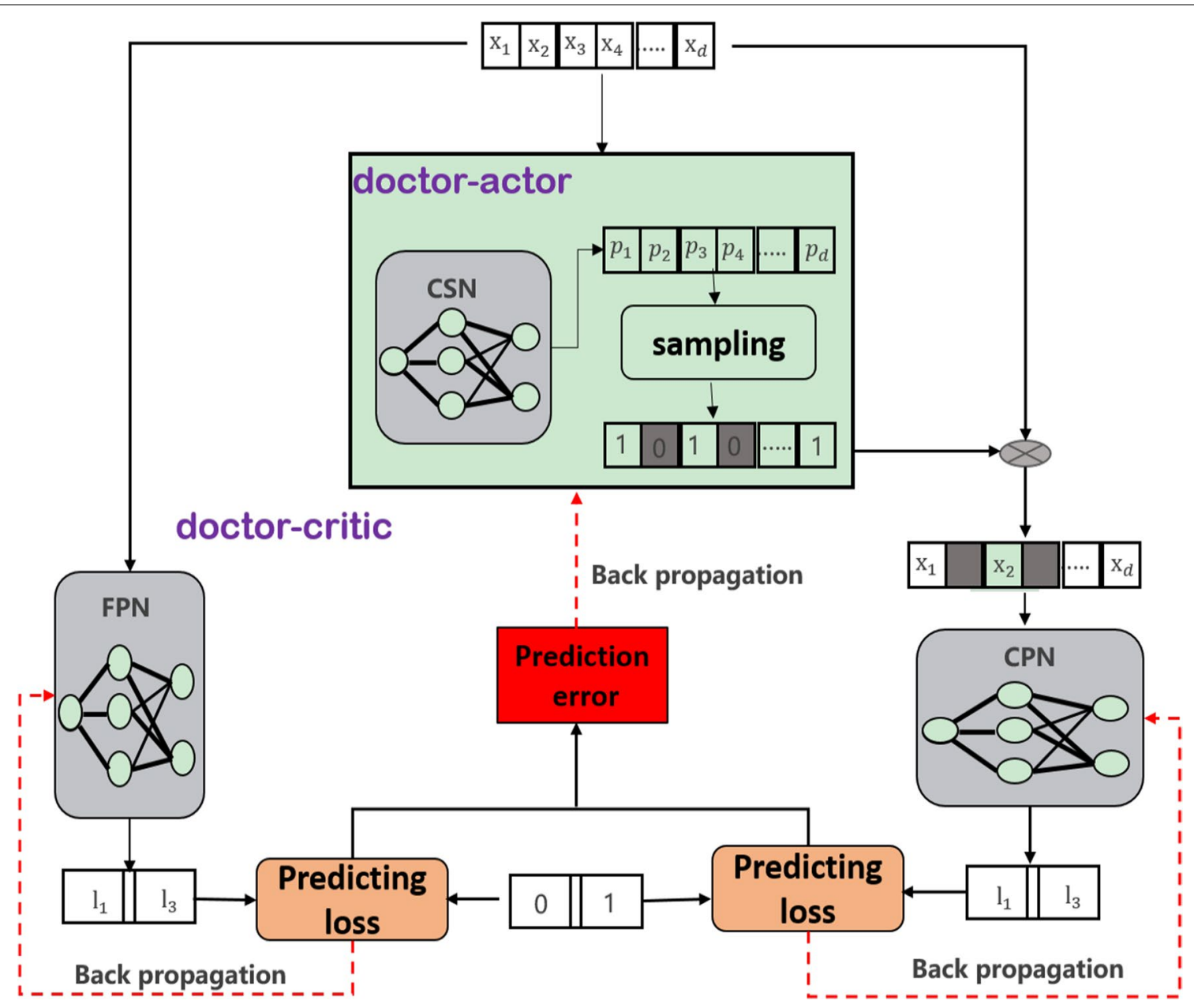

Fig. 5 The instance is input to the selector network, which outputs the selection probability vector. The selection vector is then sampled based on these probabilities. Then, the prediction network receives the selected features and makes predictions, and the baseline network gives the entire feature vector and makes predictions. Each of these networks is back-propagated training using real labels. Then subtract the loss of the baseline network from the loss of the prediction network, which is used to update the selector network. CPN counterfactual prediction network, CSN counterfactual selection network, FPN fact prediction network

model, and then we can get the optimal subset of the feature and the prediction result.

\section{Analysis of quantitative causal effects of selected features} Chattopadhyay [8] simplified the multilayer neural network into a two-layer causal structure model, and calculated the average causal effect(ACE) of input neurons on output neurons. Figure 6 . Based on this work, this section uses integral gradient to improve the calculation of the average causality effect of qualitative feature selection.

Given a neural network with input $l_{1}$ and output $l_{n}$, we hence measure the ACE of an input feature $x_{i}=\alpha \in l_{1}$ with value $\alpha$ on an output feature $y \in l_{n}$ as: (See the Additional file 1: Appendix for specific definitions)

$$
A C E_{d o\left(x_{i}=\alpha\right)}^{y}=\mathrm{E}\left[\mathrm{y} \mid \mathrm{do}\left(x_{i}=\alpha\right)\right]-\text { baseline }_{x_{i}}
$$

We define the baseline value of each input neuron as:

$$
\text { baseline }_{x_{i}}=E_{x_{i}}\left[E_{y}\left[y \mid \operatorname{do}\left(x_{i}=\alpha\right)\right]\right]
$$

In the implementation, we evaluate the baseline by evenly perturbing the input neuron $x_{i}$ from a fixed interval of $\left[\operatorname{low}_{i}, h i g h_{i}\right]$ and calculating the intervention expected value. 


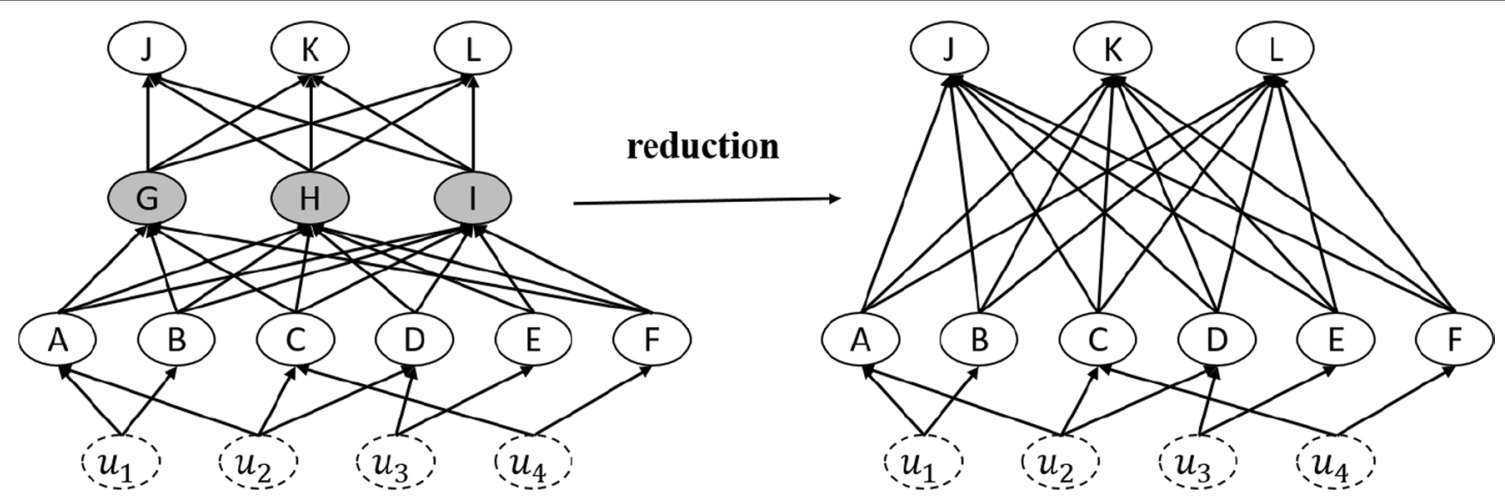

Fig. 6 Feed-forward neural network as SCM. The dotted circles represent exogenous random variables that can be used as common causes for different input feature

Consider an output neuron y in the reduced SCM $M^{\prime}\left(\left[l_{1}, l_{n}\right], U, f^{\prime}, P_{u}\right)$ obtained by marginalizing out the hidden neurons in a given neural network $M^{\prime}\left(\left[l_{1}, l_{n}\right], U, f^{\prime}, P_{u}\right)$. The causal mechanism can be written as $\mathrm{y}=f_{y}^{\prime}\left(x_{1}, x_{2} \ldots x_{k}\right)$, where $x_{i}$ refers to neuron $\mathrm{i}$ in the input layer, and $\mathrm{k}$ is the number of input neurons. If we perform a $d o\left(x_{i}=\alpha\right)$ operation on the network, the causal mechanism is given by $\mathrm{y}=f_{y \mid d o\left(x_{i=\alpha}\right)}^{\prime}\left(x_{1}, x_{2} \ldots x_{k}\right)$. Let $\mu_{j}=E\left[x_{j} \mid d o\left(x_{i}=\alpha\right)\right] \forall x_{j} \in l_{1}$. Now, the second-order Taylor's expansion of the causal mechanism $f_{y \mid d o\left(x_{i=\alpha}^{\prime}\right)}^{\prime}$ around the vector $\mu=\left[\mu_{1}, \mu_{2} \ldots \mu_{k}\right]$ is given by (recall $l_{1}$ is the vector of input neurons):

$$
f_{y}^{\prime}\left(l_{1}\right) \approx f_{y}^{\prime}(\mu)+\nabla^{T} f_{y}^{\prime}(\mu)\left(l_{1}-\mu\right)+\frac{1}{2}\left(l_{1}-\mu\right)^{T} \nabla^{2} f_{y}^{\prime}(\mu)\left(l_{1}-\mu\right)
$$

Based on the results of the first-order integral gradi-

Take expectations on both sides at the same time (marginalize other input neurons): gradient to replace the solution of the gradient in formula 14. The average result of the gradient of each point on the straight line from $x_{i}$ to $\widehat{x}_{i}$. Because we're taking into account the gradients of all the points along the path, we're no longer constrained by the fact that the gradient at one point is zero. In the implementation we chose the zero vector as the benchmark. The first-order integral gradient calculation formula is as follows:

$$
\nabla f_{y}^{\prime}(\mu)=\left|\left[\frac{1}{n} \sum_{k=1}^{n}\left(\left.\nabla_{\gamma} f_{y}^{\prime}(\gamma(a))\right|_{\gamma(a)=(1-a) x+a \widehat{x}, a=\frac{k}{n}}\right)\right][\widehat{x}-x]_{i}\right|
$$

$$
E\left[f_{y \mid d o\left(x_{i=\alpha}\right)}^{\prime}\left(\left(l_{1}\right)\right] \approx f_{y}^{\prime}(\mu)+\frac{1}{2} \operatorname{Tr} \nabla^{2} f_{y}^{\prime}(\mu) E\left[\left(l_{1}-\mu\right)^{T}\left(l_{1}-\mu\right) \mid \operatorname{do}\left(x_{i}=\alpha\right)\right]\right.
$$

We now only need to calculate the individual interventional means $\mu$ and the interventional covariance between input features $E\left[\left(l_{1}-\mu\right)^{T}\left(l_{1}-\mu\right) \mid d o\left(x_{i}=\alpha\right)\right]$ to compute formula (14). We assume that the input neuron after intervention is d-separated from all other input neurons (See Additional file 1: Appendix for details). Therefore, the intervention mean and covariance are equal to the observed mean and covariance, respectively.

The formula (14) needs to calculate the second-order Hessian matrix of $f_{y \mid d o\left(x_{i=\alpha}\right)}^{\prime}$. There is gradient saturation in the deep neural network training, and the average causal effect calculated according to formula (14) may also be saturated, that is, we don't get effective average causal effect. Therefore, we introduce the integral matrix of Formula (14) and calculate the average causal effect of input neurons on output neurons.

Therefore, combining the above two-stage model, we can perform feature selection prediction and average causal effect analysis for each patient. See the detailed experimental results in the following section.

\section{Results and experiments}

In this section, we experimentally evaluate the proposed model on synthetic data, open source data, and real world medical data. We evaluate our performance both at the relevance of feature selection and the accuracy of prediction. We compare our qualitative feature 
selection model with two methods: LIME [9], and Shapley [10].compare our prediction model with XGBOOST and LASSO regularized linear model. In order to verify the effectiveness of the model, we also compare the open source data and real medical data with neural and support vector machine (SVM).Finally, we conduct quantitative analysis on the causal effect of the selected features.

The experimental environment of this article was based on the server: Ubuntu 16.04 LTS was used as the operating system with Intel Xeon e5-2650 V4 processor and Nvidia GTX 1080 Ti GPU, the memory is 63 GB. Pytorch was used to build the model, and Python3.6 was used as the programming tool.

\section{Synthetic data experiments}

We firstly verify the effectiveness of model feature selection based on synthetic data. The input features are generated from an 11-dimensional Gaussian distribution with no correlations across the features. The label $\mathrm{Y}$ is sampled as a Bernoulli random variable with $\mathrm{P}(\mathrm{Y}=0 \mid \mathrm{X})=\frac{\operatorname{logit}(X)}{1+\operatorname{logit}(X)}$ where $\operatorname{logit}(\mathrm{X})$ is varied to create 3 different synthetic datasets:

$$
\begin{aligned}
& \text { Datasets1 : } \exp \left(X_{0} X_{1}\right) \\
& \text { Datasets2 : } \exp \left(\sum_{i=2}^{5} X_{i}^{2}-4\right) \\
& \text { Datasets3 : }-10 \times \sin 2 X_{6}+2\left|X_{7}\right|+X_{8}+\exp \left(-X_{9}\right)
\end{aligned}
$$

For each of Datasets-1 to Datasets-3 We generate 40,000 samples, 20,000 samples for training and 20,000 samples for testing. When focusing on feature selection, the performance indicators we use are true positive rate (TPR) (the higher the better) and false discovery rate (FDR) (the lower the better) to measure the performance of the method. We use the area under the receiver operating characteristic curve (AUROC), the area under the accuracy recall curve (AUPRC) and accuracy when the focus is prediction.

In this experiment we analyze the effect of using feature selection as a pre-processing step for prediction. We first perform feature selection and then train a 3-layer fully connected network to perform predictions on top of the (feature-selected) data. In this setting we compare the two feature selection methods (Lime and shapely) Furthermore, we also compare with the predictive model with XGBOOST and LASSO regularized linear model.

As demonstrated by Table 1, both TPR and FDR of our model are substantially superior to the Lime and Shapely methods. TPR and FDR of dataset 1 are $100 \%$ and 0 . TPR and FDR of dataset 2 are $100 \%$ and 0 . TPR and FDR of dataset 3 are $92 \%$ and 0 . It indicates that our method is capable of detecting relevant features. In order to verify the effectiveness of the selection features of the counterfactual prediction network, we conducted experiments based on the counterfactual prediction network (Model proposed in this paper), the Factual prediction network, XGBOOST and LASSO respectively. The experimental results are shown in the Table 2.As can be seen in Table 2, there is a significant performance improvement when discarding all of the irrelevant features. However, neither of the feature selection methods (XGBOOST and LASSO) are capable of achieving this improvement.

Figure 7 describes the causal effect analysis diagram of the dataset sample. As can be seen in Fig. 7a, the selection of $\mathrm{X} 0$ and $\mathrm{X} 1$ in our model indicates the correctness of the selection of causal features. $\mathrm{X} 0$ and $\times 1$ are positively correlated with the average causal effect of negative classification results, and vice versa. The attribution curve exactly fits the data generation process. Figure $7 \mathrm{~b}$ also shows the attribution process. From the data generation formula (17), we can see that when $\mathrm{X}<0$, the probability

Table 2 Prediction performance results

\begin{tabular}{|c|c|c|c|c|c|c|}
\hline \multirow{2}{*}{$\begin{array}{l}\text { Dataset } \\
\text { Metrics (\%) }\end{array}$} & \multicolumn{2}{|c|}{ Dataset1 } & \multicolumn{2}{|c|}{ Dataset2 } & \multicolumn{2}{|c|}{ Dataset3 } \\
\hline & TPR & FDR & TPR & FDR & TPR & FDR \\
\hline Our model & 100 & 0 & 100 & 0 & 92 & 0 \\
\hline LIME & 13.8 & 86.2 & 100 & 0 & 98.1 & 1.9 \\
\hline Shapley & 60.4 & 39.6 & 93.3 & 6.7 & 65.2 & 9.1 \\
\hline
\end{tabular}

\begin{tabular}{ccccl}
\hline Dataset & XGBOOST & With LASSO & $\begin{array}{l}\text { Factual } \\
\text { prediction } \\
\text { network }\end{array}$ & $\begin{array}{l}\text { Counterfactual } \\
\text { prediction } \\
\text { network }\end{array}$ \\
\hline AUROC & & & & \\
Dataset1 & $.574 \pm 0.10$ & $.498 \pm 0.06$ & $.681 \pm 0.02$ & $.693 \pm 0.06$ \\
Dataset2 & $.872 \pm 0.03$ & $.823 \pm 0.61$ & $.864 \pm 0.61$ & $.877 \pm 0.03$ \\
Dataset3 & $.899 \pm 0.01$ & $.862 \pm 0.03$ & $.890 \pm 0.03$ & $.911 \pm 0.02$ \\
AUPRC & & & & \\
Dataset1 & $.577 \pm 1.02$ & $.499 \pm 0.08$ & $.681 \pm 0.04$ & $.694 \pm 0.03$ \\
Dataset2 & $.878 \pm 0.31$ & $.591 \pm 0.37$ & $.861 \pm 0.21$ & $.886 \pm 0.04$ \\
Dataset3 & $.904 \pm 0.04$ & $.890 \pm 0.02$ & $.890 \pm 0.05$ & $.905 \pm 0.02$ \\
\hline
\end{tabular}

Table 1 Feature selection result for synthetic datasets 

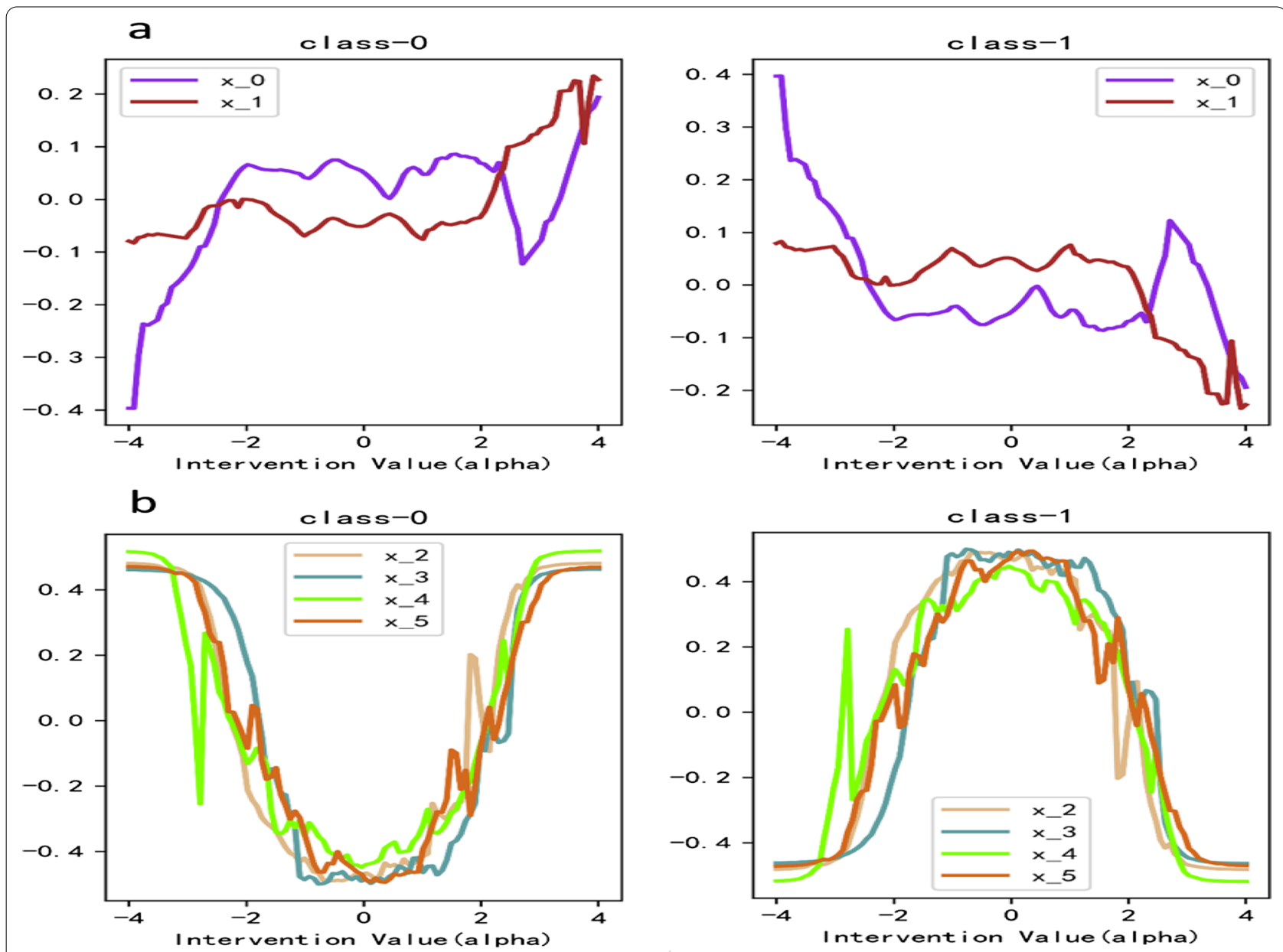

Fig. 7 Causal effect analysis diagram. a Datasets1 and b Datasets2

of a sample being classified as negative is monotonically decreasing, and when $x>0$, the probability of being classified as negative is monotonically increasing. The figure clearly describes that the model chooses $\times 2, \times 3, \times 4$, and $\times 5$ as prediction features. Interfering with these four feature values, the corresponding causal effects are consistent with the monotonicity of the data generation process, indicating the effectiveness of the model designed in this paper for the quantitative analysis of causal effects. It can also be seen that the model captures the causal relationship between each variable and Y well. Although the model chooses the variable $\times 9$, it can be seen that the average causal effect of $\times 9$ on $y$ is basically 0 . It shows that the variable $\times 9$ has no causality with the prediction task.

\section{Obesity levels based on eating habits and physical condition data set}

In this section we use open source healthcare data to perform a series of further experiments. This dataset include data for the estimation of obesity levels in individuals from the countries of Mexico, Peru and Colombia, based on their eating habits and physical condition. The data contains 17 attributes and 2111 records. $77 \%$ of the data was generated synthetically using the Weka tool and the SMOTE filter, $23 \%$ of the data was collected directly from users through a web platform. All data was labeled and the class variable was created with the values of: normal and abnormal in this experiment (See the Additional file 1: Appendix for the specific attributes of the data set).

Table 3 Prediction performance results

\begin{tabular}{lllll}
\hline Datasets & Method & AUROC & AUPRC & ACC \\
\hline Obesity & XGBOOST & $0.898 \pm 0.04$ & $0.915 \pm 0.02$ & $0.855 \pm 0.06$ \\
& LRWith LASSO & $0.840 \pm 0.05$ & $0.92 \pm 0.03$ & $0.834 \pm 0.01$ \\
& Neural network & $0.839 \pm 0.02$ & $0.89 \pm 0.01$ & $0.831 \pm 0.01$ \\
& SVM & $0.810 \pm 0.01$ & $0.83 \pm 0.02$ & $0.82 \pm 0.02$ \\
& With our model & $0.840 \pm 0.04$ & $0.900 \pm 0.02$ & $0.836 \pm 0.06$ \\
\hline
\end{tabular}


It can be seen from Table 3 that our proposed model is basically consistent with the performance of the full feature prediction method in terms of health prediction ability. The reason for our analysis may be that the number of features is inherently small and there is a strong correlation between the selected features and the predicted labels, so the advantages of our feature selection model have not been reflected. In addition, in the experiment, we drew a heat map of the feature selection probability of test patients. Figure 8 shows that the main reason for the model to predict patients is weight, FHWO, CAEC and FAF variables.

Figure 9a, b depict average causal effect for the two classes and selected features. These plots easily reveal that smaller weight is positively causal $(A C E \geq 0)$ for Normal class and negatively causal $(\mathrm{ACE}<0)$ for Abnormal class. Consumption of food between meals (CAEC) is a discrete value (No:0, Sometimes:1, Frequently:2, Always:3). It can be easily seen from the figure that frequently Consumption of food between meals is negatively causal for normal class and positively causal for Abnormal class. Therefore, from the results of causal effect analysis, the conclusions of the model are consistent with common medical knowledge.

\section{Heart failure data}

In this section, we use heart failure datasets to perform a series of further experiments. The data has 1452 patients each with 84 measured features, which were collected from surgery patient in hospital (the First Affiliated Hospital of Military Medical University of the Army) of china from 2014 to 2018.The label is heart failure. The age, gender and label distribution were shown in Fig. 10 (See the Additional file 1: Appendix for the specific attributes of the data set).

As can be seen in Table 4, there is a slight performance improvement when discarding all of the irrelevant features. However, we can get which features the model prediction focuses on from the feature selection probabilistic heat map. Figure 11 depicts a heat map of the average probability of features selected for heart failure in male and female patients. It is concluded from the map that the male and female models focus on the same features.

Figure 12 depicts the causal effect of patient selection feature. As we can see from the figure that when the patient value is in the middle, the causal effect on the prediction of heart failure is not obvious. Because the value is in the normal range. When the patient's value is at both ends, the causal effect value changes

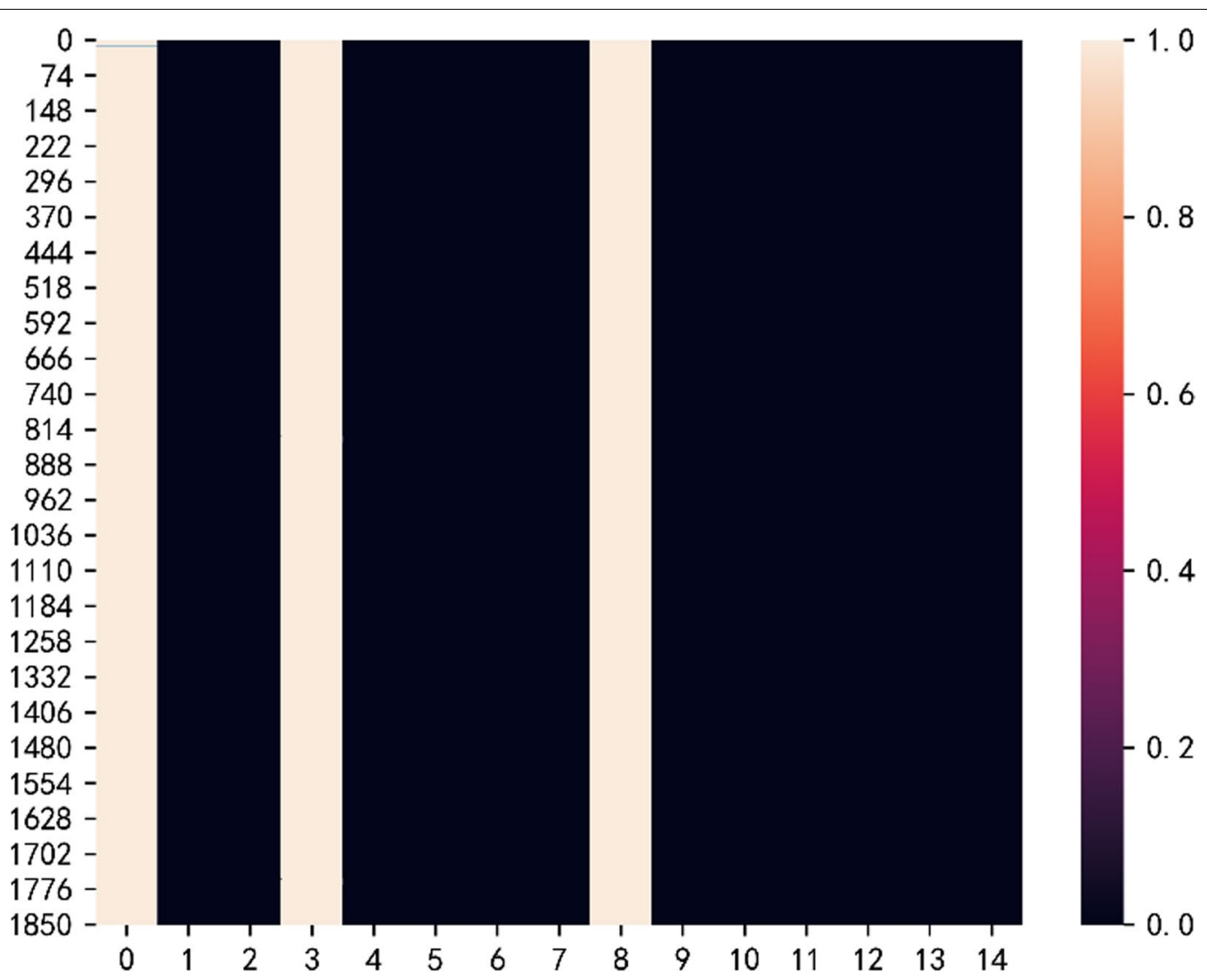

Fig. 8 Feature selection probabilistic heat map 

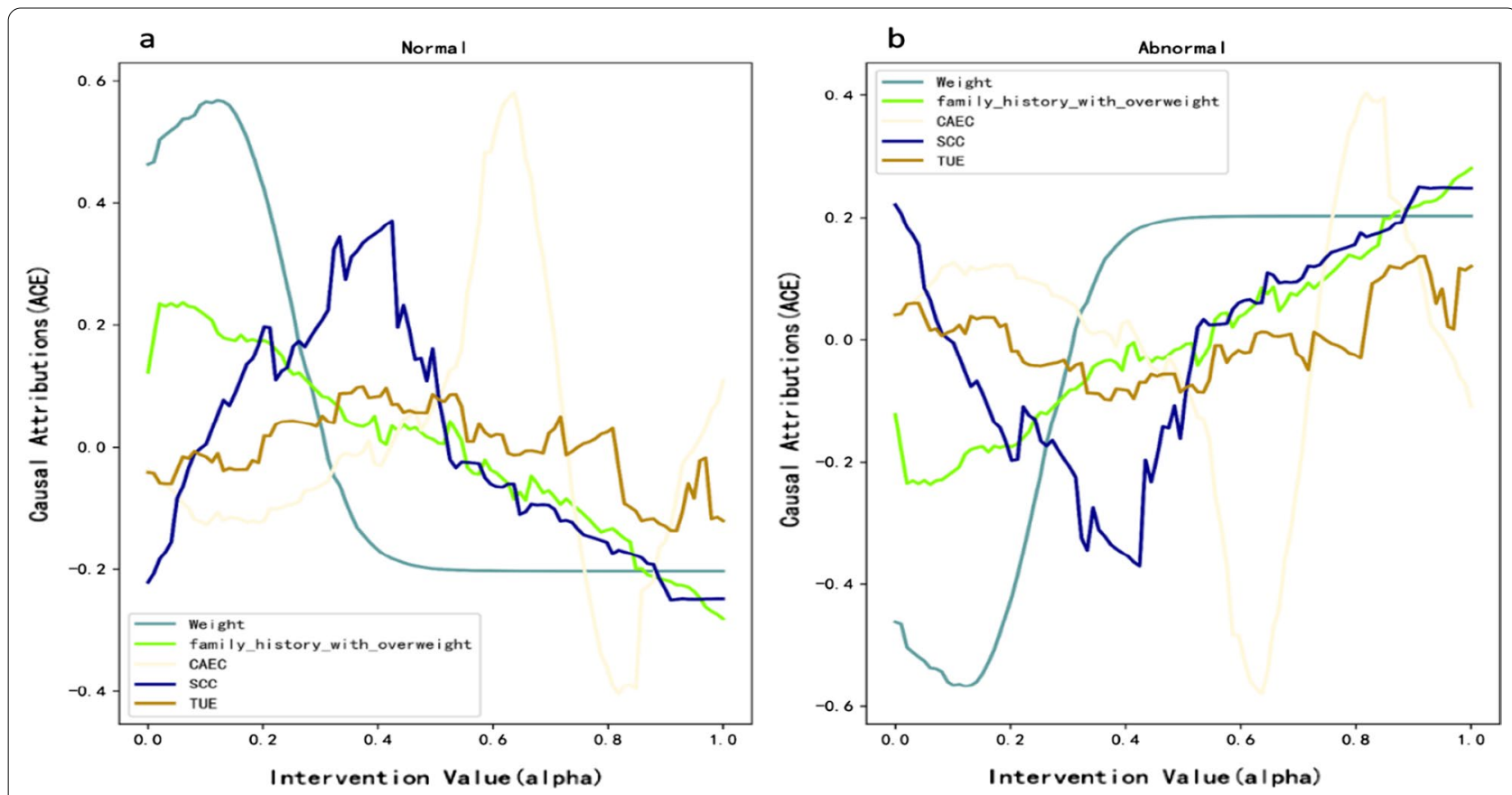

Fig. 9 Causal effect analysis diagram. a Normal and $\mathbf{b}$ abnormal
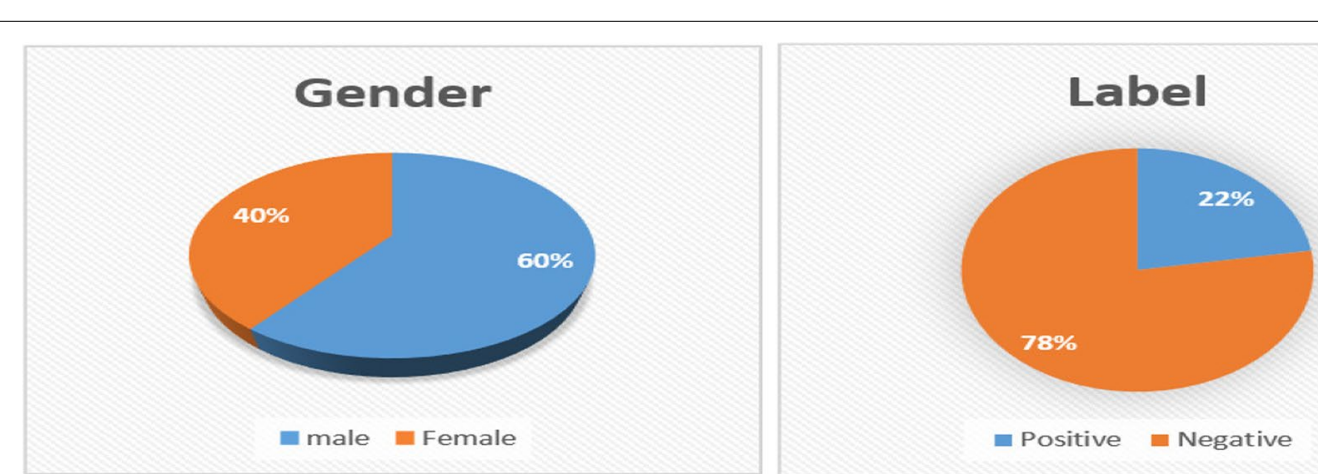

a

Age Distribution

b

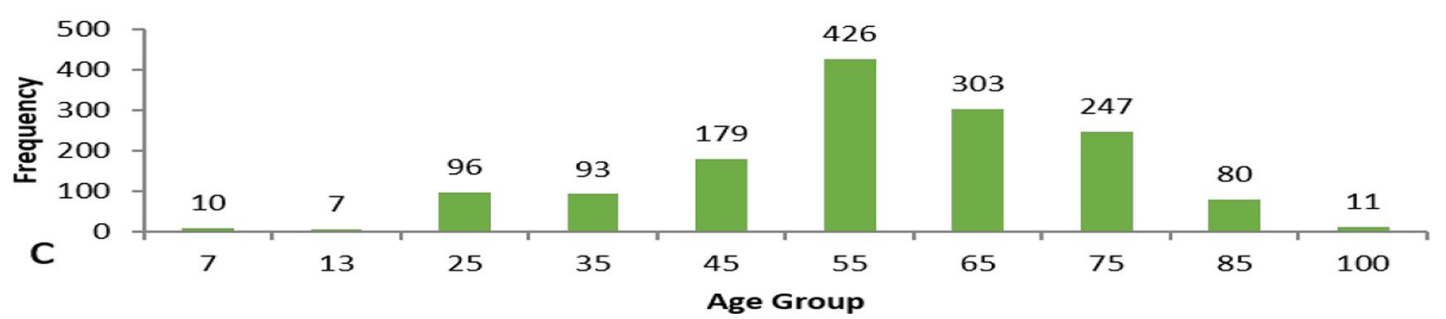

Fig. 10 Heart failure data set distribution. a Gender, b label, c age

significantly. In particular, the variables $\mathrm{x} \_13, \mathrm{x} \_28$, $\mathrm{x} \_32, \mathrm{x} \_57$ have a greater impact on the prediction of the patient. $x \_13$ is the Direct bilirubin (DBIL). $x \_28$ is the patient's intraoperative pulse variance. $x \_32$ is the variance of the patient's intraoperative spo2. $x \_57$ is the variance of the patient's intraoperative heart rate.
The figure reveal that the larger $\mathrm{x} \_28, \mathrm{x} \_32$ and $\mathrm{x} \_57$ are positively causal $(\mathrm{ACE} \geq 0)$ for heart failure. The analysis of the model is consistent with common medical knowledge. In addition, patient's direct bilirubin is also positively causal for heart failure. We analyzed 
Table 4 Prediction performance results

\begin{tabular}{lllll}
\hline Datasets & Method & AUROC & AUPRC & ACC \\
\hline Heart failure & XGBOOST & $0.90 \pm 0.04$ & $0.792 \pm 0.02$ & $0.870 \pm 0.06$ \\
& LRWith LASSO & $0.91 \pm 0.03$ & $0.723 \pm 0.02$ & $0.90 \pm 0.11$ \\
& Neural network & $0.912 \pm 0.02$ & $0.791 \pm 0.02$ & $0.899 \pm 0.01$ \\
& SVM & $0.881 \pm 0.01$ & $0.781 \pm 0.02$ & $0.851 \pm 0.02$ \\
& With our model & $0.924 \pm 0.04$ & $0.808 \pm 0.02$ & $0.90 \pm 0.06$ \\
\hline
\end{tabular}

that the patient may have liver disease, which can lead to heart problems.

\section{Discussion}

Traditional interpretability mainly focuses on statistical interpretability, while causal interpretability aims to answer questions related to causal intervention interpretability and counterfactual interpretability. For instance, traditional machine interpretability frameworks are not capable to answer causal questions such as "What is the impact of the nth filter of the mth layer of a deep neural network on the predictions of the model?" which are helpful and required for understanding a neural network model. Chattopadhyay et al. [8] propose an attribution method based on the first principle of causality. The proposed framework models the structure of the machine learning algorithm as an SCM. It then proposes a scalable causal inference approach to the estimate individual treatment effect of a desired component on the decision made by the algorithm. Therefore, we propose a two-stage prediction method (instance feature selection prediction and causal effect analysis) for instance disease prediction base on this work. The results of our experiments on synthetic data, open source data and real medical data show that our proposed method can provide qualitative and quantitative causal explanations for the model while giving prediction results.

The limitation of this work is that we only focus on the static attribute data of patients, while the model cannot deal with the clinical time series data. Future work will include extending to apply in the temporal setting. One such avenue of exploration for this would be to replace each of the networks with an RNN. This method can apply to medical time series data. Importantly, we believe this work can encourage viewing medical and health issues from a causal lens, and answering further causal questions such as: which counterfactual questions might be asked and answered in a medical and health issues, can a causal chain exist in medical and health issues and so on.

\section{Conclusions}

This work presented a new causal perspective to feature selection and prediction. We propose a two-stage prediction method for instance disease prediction. Firstly, qualitative feature selection is performed on patients. The method is based on counterfactual and uses a reinforcement learning framework to design an interpretable instance feature selection prediction model. The methods of quantitative feature analysis views a neural

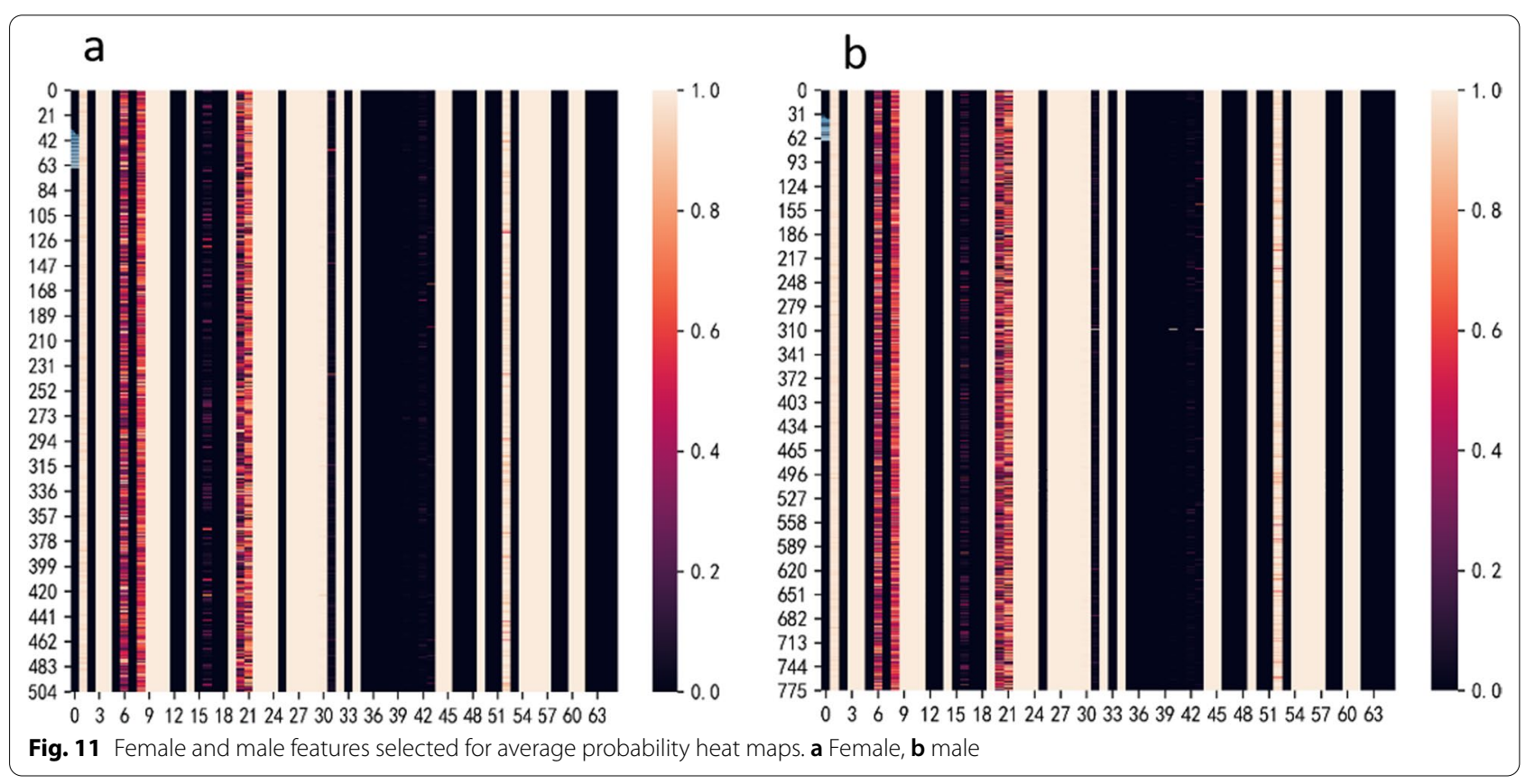




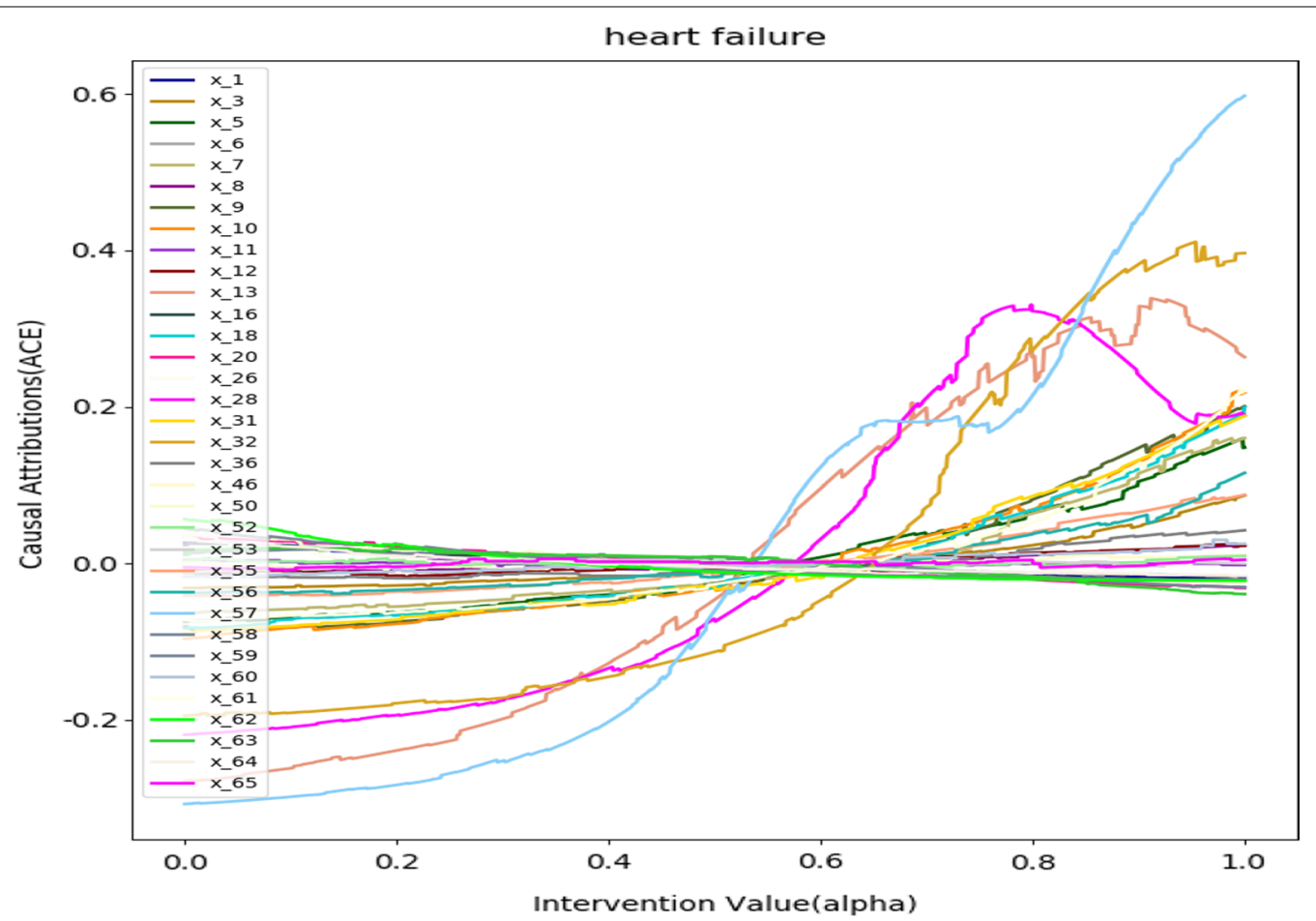

Fig. 12 Average causal effect in patients with heart failure

network as an Structural Causal Model (SCM)to calculate the Average Causal Effect (ACE) of selected features in neural networks. The experiments on synthetic, open source, and real data show that the method can effectively select patient attributes for prediction and elicit causal effect of input on output data in neural networks.

\section{Abbreviations}

ACE: Average causal effect; SCM: Structural causal model; Al: Artificial intelligence; SVM: Support vector machine; FCBF: Fast correlation-based filter; MRMR: Minimum redundancy and maximum relevance.

\section{Supplementary Information}

The online version contains supplementary material available at https://doi. org/10.1186/s12911-022-01788-8.

Additional file 1. Neural Network Attribution Related Definition. Definition of TPR and FDR. Data Set Attributes.

\section{Acknowledgements}

Not applicable.

\section{Authors' contributions}

$Y C$ conceived the study and performed the experiments. $Y C$ and JZ wrote the paper and have drafted the work or substantively revised it. XLQ reviewed and edited the manuscript. All authors read and approved the manuscript.

\section{Funding}

This work is supported by the National Key Research and Development Plan of China (2018YFC0116704), which provides environmental and financial support in data collection. In addition, it is supported by youth innovation promotion association of the Chinese Academy of Sciences (2020377), which provides technical consultation and financial support in model design and experiment.

\section{Availability of data and materials}

The data of this experiment comes from three parts: synthetic data, open source data and real medical data. Synthetic data is automatically generated by computer based on formula. Obesity levels based on eating habits and physical condition Data Set came from the kaggle competition. It can be downloaded from the website (https://www.kaggle.com/ankurbajaj9/obesi ty-levels). The real medical data of patients with heart failure comes from the cooperative unit (Southwest Hospital).The raw data required to reproduce these findings cannot be shared at this time as the data also forms part of an ongoing study. If someone wants to reasonable request the data, you may contact the corresponding author.

\section{Declarations}

\section{Ethics approval and consent to participate}

The procedures followed in this study strictly comply with the ethical standards formulated by the ethics committee of Southwest Hospital of the Third Military Medical University (Chongqing, China).This study was approved by the Ethics Committee of the Southwest Hospital of Third Military Medical University and the Approved No. of ethic committee is KY201936.All participants voluntarily participated in the study and signed the informed consent.

\section{Consent for publication}

Not applicable.

\section{Competing interests}

The authors declare that they have no competing interests. 


\section{Author details}

${ }^{1}$ Chengdu Institute of Computer Applications, Chinese Academy of Sciences, Chengdu, China. ${ }^{2}$ Chongqing Institute of Green and Intelligent Technology, Chinese Academy of Sciences, Chongqing, China. ${ }^{3}$ University of Chinese Academy of Sciences, Beijing, China.

Received: 1 March 2021 Accepted: 18 February 2022 Published online: 26 February 2022

\section{References}

1. David C, et al. DNA methylation-based classification of central nervous system tumours. Nat Int Wkly J Sci. 2018;555(7697):469-74.

2. Liu Y, et al. Detecting cancer metastases on gigapixel pathology images. arXiv preprint arXiv:1703.02442 (2017).

3. Varun $\mathrm{G}$, et al. Development and validation of a deep learning algorithm for detection of diabetic retinopathy in retinal fundus photographs. JAMA. 2016;316(22):2402-10.

4. Pearl J. Causality. Cambridge: Cambridge University Press; 2009.

5. Peters J, Janzing D, Schölkopf B. Elements of causal inference: foundations and learning algorithms. Cambridge, MA: MIT Press; 2017.

6. Reichenbach $\mathrm{H}$. The direction of time, vol. 65. Berkeley: University of California Press; 1991.

7. Angrist JD, Imbens GW, Rubin DB. Identification of causal effects using instrumental variables. J Am Stat Assoc. 1996;91(434):444-55.

8. Chattopadhyay A, et al. Neural network attributions: a causal perspective. In: International conference on machine learning. PMLR (2019).

9. Ribeiro MT, Singh S, Guestrin C. "Why should i trust you?" Explaining the predictions of any classifier. In: Proceedings of the 22nd ACM SIGKDD international conference on knowledge discovery and data mining (2016).

10. Chen $X$, et al. Infogan: interpretable representation learning by information maximizing generative adversarial nets. arXiv preprint arXiv:1606. 03657 (2016).

11. Goodfellow I, et al. Deep learning, vol. 1. Cambridge: MIT Press; 2016.

12. Deng L, Yu D. Deep learning: methods and applications. Found Trends Signal Process. 2014;7(3-4):197-387.

13. Gilmer J, et al. Neural message passing for quantum chemistry. In: International conference on machine learning. PMLR (2017).

14. Veličković P, et al. Graph attention networks. arXiv preprint arXiv:1710. 10903 (2017)

15. Vaswani A, et al. Attention is all you need. arXiv preprint arXiv:1706.03762 (2017).

16. Bahdanau D, Cho K, Bengio Y. Neural machine translation by jointly learning to align and translate. arXiv preprint arXiv:1409.0473 (2014).

17. Jolliffe I. Principal component analysis. Technometrics. 2003;45(3):276.

18. Hyvärinen A, Oja E, Neural Networks Research Centre. Independent component analysis: algorithms and applications. Neural Netw. 2000;13(4):411-30.

19. Von Luxburg U. A tutorial on spectral clustering. Stat Comput. 2007;17(4):395-416.

20. Kingma DP, Welling M. Auto-encoding variational bayes. arXiv preprint arXiv:1312.6114 (2013).

21. Higgins I., et al. beta-VAE: Learning Basic Visual Concepts with a Constrained Variational Framework. in ICLR. 2017.

22. Simonyan K, Vedaldi A, Zisserman A. Deep inside convolutional networks: visualising image classification models and saliency maps. arXiv preprint arXiv:1312.6034 (2013).

23. Zeiler MD, Fergus R. Visualizing and understanding convolutional networks. In: European conference on computer vision. Springer (2014).

24. Springenberg JT, et al. Striving for simplicity: the all convolutional net. arXiv preprint arXiv:1412.6806 (2014).

25. Ramprasaath RS, et al. Grad-CAM: visual explanations from deep networks via gradient-based localization. Int J Comput Vis. 2020;128(2):336-59.

26. Adebayo J, et al., Sanity checks for saliency maps. arXiv preprint arXiv: 1810.03292 (2018).

27. Ghorbani A, Abid A, Zou J. Interpretation of neural networks is fragile. In: Proceedings of the AAAl conference on artificial intelligence (2019).

28. Goyal Y, et al. Counterfactual visual explanations. In: International conference on machine learning. PMLR (2019).
29. Kommiya Mothilal R, Sharma A, Tan C. Explaining machine learning classifiers through diverse counterfactual explanations. arXiv e-prints arXiv: 1905.07697 (2019).

30. Harradon M, Druce J, Ruttenberg B. Causal learning and explanation of deep neural networks via autoencoded activations. arXiv preprint arXiv: 1802.00541 (2018).

31. Narendra T, et al. Explaining deep learning models using causal inference. arXiv preprint arXiv:1811.04376 (2018).

32. Pearl J. Theoretical impediments to machine learning with seven sparks from the causal revolution. arXiv preprint arXiv:1801.04016 (2018).

33. Yu L, Liu H. Efficient feature selection via analysis of relvance and redundancy. J Mach Learn Res. 2004;5(12):1205-24.

34. Sakar CO, Kursun O, Gurgen F. A feature selection method based on kernel canonical correlation analysis and the minimum redundancymaximum relevance filter method. Expert Syst Appl. 2012;39(3):3432-7.

35. Brown G, Pocock A, Zhao MJ, et al. Conditional likelihood maximisation: a unifying framework for information theoretic feature selection. J Mach Learn Res. 2012;13(1):27-66.

\section{Publisher's Note}

Springer Nature remains neutral with regard to jurisdictional claims in published maps and institutional affiliations.

Ready to submit your research? Choose BMC and benefit from:

- fast, convenient online submission

- thorough peer review by experienced researchers in your field

- rapid publication on acceptance

- support for research data, including large and complex data types

- gold Open Access which fosters wider collaboration and increased citations

- maximum visibility for your research: over 100M website views per year

At BMC, research is always in progress.

Learn more biomedcentral.com/submissions 